\title{
An Islet-Cell Protein Tyrosine Phosphatase Is a Likely Precursor to the 37-kDa Autoantigen in Type 1 Diabetes: Human and Macaque Sequences, Tissue Distribution, Unique and Shared Epitopes, and Predictive Autoantibodies
}

\author{
James LaGasse,* Laura Jelinek, ${ }^{\dagger}$ Shannon Sexson, ${ }^{\dagger}$ \\ Cathy Lofton-Day, ${ }^{+}$John Breininger,* Paul Sheppard, ${ }^{+}$ \\ Wayne Kindsvogel, ${ }^{\dagger}$ and William A. Hagopian* \\ *Department of Medicine, University of Washington, Seattle, \\ Washington, U.S.A. \\ 'ZymoGenetics, Inc., Seattle, Washington, U.S.A.
}

\begin{abstract}
Background: We sought to identify novel islet-cell autoantigens to better understand the pathogenesis, prediction, and immunotherapy of type 1 diabetes.

Materials and Methods: Macaque and human islet cDNA libraries expressed in mammalian cells were screened with human diabetes sera. A positive clone was sequenced directly and after $5^{\prime}$ rapid amplification of cDNA ends (RACE). Northern blotting and in situ hybridization revealed the tissue distribution of the corresponding protein. Antigen, expressed by in vitro translation, and tryptic peptides were analyzed by SDS-PAGE. For the immunoprecipitations, 183 diabetic, 60 prediabetic, and 91 control sera were used. Truncated antigens were used in immunoprecipitations for epitope mapping. Recombinant antigen expressed in transfected fibroblasts was used in competition assays.

Results: Sequencing yielded an 111-kDa, 1,013 amino acid, transmembrane protein (M1851) containing con-
\end{abstract}

sensus protein tyrosine phosphatase (PTPase) sequence. M1851 was $77 \%$ identical in the intracellular domain, but only $31 \%$ identical extracellularly, to the islet-cell autoantigen ICA512. mRNA localized to brain, prostate, pancreatic islets, and adrenal medulla. After limited trypsinization, the in vitro translated antigen was 37 $\mathrm{kDa}$. M1851 was recognized by $47 \%$ of prediabetes sera, $31 \%$ of new diabetes sera, but only $1 \%$ of healthy controls. Only $1 / 73$ sera binding M1851 failed to bind ICA512, whereas $42 / 114$ binding ICA512 did not bind M1851. M1851 reactivity was not fully displaced by ICA512 in 24/49 sera. Removing the C-terminal 27, 80, or 160 amino acids of $\mathrm{Ml} 851$ decreased reactivity by $70 \%, 90 \%$, and $100 \%$, respectively.

Conclusions: This new islet-cell PTPase is likely to be the precursor to the $37-\mathrm{kDa}$ tryptic fragment antigen. It is structurally related to ICA512 but has distinct diabetes autoantibody epitopes located at the $\mathrm{C}$ terminus.

\section{INTRODUCTION}

Insulin-dependent diabetes is an organ-specific autoimmune disease resulting from aberrant im-

Address correspondence and reprint requests to: William Hagopian, Department of Medicine, Box 357710, University of Washington, Seattle, WA 98195, U.S.A. Phone: 206-6854775; Fax: 206-543-3169; E-mail: wah@u.washington.edu

The macaque sequence reported in this paper has been deposited in the GenBank data base (accession no. U91574). mune response to specific $\beta$-cell autoantigens (1). Humoral autoimmunity in type I diabetes (IDDM) is generally described in terms of the classic islet-cell antigen (ICA) assay (2) using frozen sections of human pancreas as assay substrate. Serum autoantibodies are detected using a fluorescent second antibody and indirect fluorescence microscopy. Multiple antigens are targets, including glutamic acid decarboxylase (GAD) (3) and the putative protein tyrosine phosphatase 
(PTPase) ICA512 (4). Of new IDDM sera, 28/969 had ICA reactivity but were negative for autoantibodies to GAD, insulin, and ICA512 (W.A. Hagopian, unpublished observation), which suggests that other antigens are also recognized by islet-cell autoantibodies.

Identification of these additional $\beta$-cell antigens could greatly improve IDDM prediction, especially in those patients without a diabetic relative, where most new cases occur. The general population has a low prevalence of IDDM, so high test specificity is critical in achieving predictive values sufficient for clinical immunotherapy. Use of independent, recombinant antigens in radiobinding assays has proven to be highly specific. For example, combining GADab and insulin autoantibodies (IAA) led to a $99.7 \%$ specificity (5). However, only $41 \%$ of the subjects were positive for both tests. Adding additional independent antibody tests, and then requiring at least two to be positive, should preserve specificity but improve sensitivity, so that more prediabetics can participate in intervention trials. Identification of $\beta$-cell autoantigens in IDDM also has an important role in potential antigen-based immunointervention therapy. Proposed therapies include vaccination, intravenous monomeric antigen or altered peptide ligands, oral tolerance, and peptide-HLA constructs (6).

One important but elusive antigen has been the precursor to the 37-kDa tryptic fragment first described by Christie et al. (7). Although the parent antigen to this tryptic fragment ran as a diffuse band at about $64 \mathrm{kDa}$ on SDS-PAGE, it was distinct from GAD (8). Presence of autoantibodies to the 37-kDa fragment were shown to be highly predictive of progression to clinical IDDM in both polyendocrine patients (9) and in first-degree relatives of current diabetic patients (10). The combination of $37-\mathrm{kDa}$ reactivity and GAD reactivity was especially predictive $(11,12)$.

To find new $\beta$-cell protein antigens such as the precursor to the $37-\mathrm{kDa}$ antigen, we constructed a highly representative islet cDNA library from pure isolated macaque islets (13). Unlike rodent islets, they are highly comparable to human islets in antigen expression (13) and have highly homologous sequences (14) of sufficient similarity to be useful in designing genespecific primers for amplifications from human insulinoma cDNA template. The macaque library was constructed in a vector optimized for highlevel transient expression in mammalian cells (15) favoring appropriate post-translational processing. Finally, screening utilized IgG from
IDDM sera carefully selected for high-titer ICA not attributable to known islet antigens. New islet antigens detected in this way were expected to be disease-relevant.

A reactive clone (M1851) had a sequence similar but distinct from the putative PTPase ICA512 $(16,17)$, also known as IA-2 (18). ICA512 is itself highly recognized by IDDM sera (19). We here define the structure of M1851, its tissue distribution, antigenic properties, and predictive role in IDDM.

\section{MATERIALS AND METHODS}

Highly purified islets were isolated from Macaca nemestrina (pigtail macaque) pancreata by collagenase digestion and density gradient centrifugation as described (13). Purified mRNA from $10^{5}$ islets was used to construct a library representative of message at all abundance levels using techniques optimizing synthesis of full-length cDNA (15). cDNA were ligated into the vector pZCEP (15) containing a colEl site and an SV40 promoter, for bacterial replication and mammalian expression, respectively. The library was electroporated into Escherichia coli $\mathrm{DH} 10 \mathrm{~B}$ cells (Gibco BRL, Gaithersburg, MD), selected on agar plates containing ampicillin, harvested, and split into 100 pools each of $10^{5}$ recombinants each for storage as glycerol stocks at $-80^{\circ} \mathrm{C}$. Pools were grown separately on agar plates containing ampicillin prior to purification of plasmid DNA by phenol-chloroform extraction.

Sera from new-onset IDDM patients with ICA titer above 50 JDFU were adsorbed with excess recombinant human GAD65 (20), insulin (NovoNordisk, Bagsvaerd Denmark), and ICA512 (kind gift of Dr. D. Rabin). Two adsorbed sera had ICA titers remaining over $50 \mathrm{JDFU}$. IgG was purified using protein A-Sepharose (Zymed, South San Francisco, CA), precleared over formalin-fixed COS-7 cells, and stored at $-80^{\circ} \mathrm{C}$ until use.

COS-7 cells grown to $70 \%$ confluence on glass slides were transfected with $1 \mu \mathrm{g}$ of pool plasmid DNA using Lipofectamine (Gibco BRL). After growth for a further $72 \mathrm{hr}$, cells were fixed and permeabilized with equal parts ethanol and acetone. Slides were incubated overnight at $25^{\circ} \mathrm{C}$ with human diabetes IgG $(2 \mathrm{mg} / \mathrm{ml})$, washed, developed using biotinylated rabbit anti-human IgG, streptavidin-gold, and silver enhancer (Amersham, Arlington Heights, IL), and screened by microscopy. Three of 100 pools were positive, 
with one or more single cells stained in each of three experiments.

The glycerol stock representing one of the positive pools was plated onto agar containing ampicillin. Colonies were probed with ${ }^{32} \mathrm{P}$-labeled cDNA coding for the intracellular domain of ICA512 obtained by polymerase chain reaction (PCR) from human glioblastoma (18). Isolated plasmid DNA from a positive colony was sequenced, yielding a sequence similar but not identical to human ICA512 $(17,18)$. Using plasmid pools from the original macaque library, a vector-specific upstream primer, and gene-specific antisense primers $5^{\prime}$-CTCTGTGGTCCATGCCTTG C-3' and 5'-GCGCGATGAACTTGGTGGAGTCTT CTTGCTCCGCCTGA-3', the remaining $5^{\prime}$ sequence was obtained by the PCR technique termed $5^{\prime}$ RACE (rapid amplification of cDNA ends) (21) (Marathon, Clontech, Palo Alto, CA). Using cDNA previously synthesized from human insulinoma mRNA, and primers based on the macaque sequence, 2,456 bases of the human homologue were also amplified by PCR and sequenced.

The second 5' RACE primer above is based on M1851 antisense sequence, just external to the transmembrane region, which is distinct between M1851 and ICA512. This oligonucleotide probe was radiolabeled with $\gamma^{32} \mathrm{P}$-ATP using T4 polynucleotide kinase (Gibco BRL). Northern analysis of RNA from 23 human tissues distributed over 3 commercially prepared blots (Clontech) each separately hybridized once. Ten milliliters of fresh Express-Hyb buffer (Clontech) containing $5 \times 10^{6} \mathrm{cpm} / \mathrm{ml}$ of labeled probe was incubated with the blots overnight at $37^{\circ} \mathrm{C}$. Blots were then washed with $6 \times$ SSC, $0.1 \%$ SDS at $25^{\circ} \mathrm{C}, 50^{\circ} \mathrm{C}$, and $60^{\circ} \mathrm{C}$, and with $2 \times \mathrm{SSC}, 0.05 \%$ SDS at $68^{\circ} \mathrm{C}$, prior to autoradiography.

The same antisense probe, as well as the corresponding ICA512 antisense probe (5'-AGC TGCCTCCTCCCTCTGTCCCACTCCTGTCTGCAA GA-3') were separately hybridized in situ with $14-\mu \mathrm{m}$ frozen sections from macaque pancreas, adrenal gland, and muscle. Sections fixed in $4 \%$ paraformaldehyde were acetylated with acetic anhydride and delipidated in chloroform prior to use. Probes were end labeled with ${ }^{33} \mathrm{P}$-dATP (New England Nuclear) using terminal deoxytransferase (Gibco BRL). Probes $(2 \mathrm{pmol} / \mathrm{ml}$ ) were incubated on sections overnight at $45^{\circ} \mathrm{C}$, washed twice in $1 \times \mathrm{SSC}$ at $60^{\circ} \mathrm{C}$ for $30 \mathrm{~min}$, dehydrated in ethanol, and apposed to autoradiography film (Hyperfilm Betamax, Amersham) for 2-6 days. In situ hybridizations were per- formed three times on each tissue with similar results.

Using primers 5 -CCACCATGCGCCATAGCT CTCAGCACAGGCTGAA-3' (sense) and 5'-TCA CTGGGGCAGGGCCTTGAG-3' (antisense) based on the human sequence, the complete 1,131nucleotide sequence of intracellular M1851 was amplified from human insulinoma cDNA, ligated into vector pcDNAII (Invitrogen, San Diego, CA), and confirmed by sequencing in both directions. Coupled transcription and translation (TNT, Promega, Madison, WI) proceeded in the presence of ${ }^{35} \mathrm{~S}$-methionine. Limited trypsinization (Sigma, St. Louis, MO) was performed at $4^{\circ} \mathrm{C}$ for 15 min by the method of Christie et al. (7).

Immunoassays each used $4 \mu$ l of human serum in duplicate incubated overnight at $4^{\circ} \mathrm{C}$ with radiolabeled icM1851, precipitated with protein A-Sepharose, washed, counted by scintigraphy, and expressed as an M1851 index = (unknown negative control)/(positive control - negative control). The positive control serum is the world ICA standard used to calculate JDF units. This serum immunoprecipitates GAD, ICA512, and M1851 (5,22). Serum samples included 91 healthy control sera (median age 22 years, $51 \%$ females), 183 unselected type 1 diabetic patients sampled at onset (median age 11 years, $49 \%$ females), and 60 type 1 diabetic patients sampled a mean of 2.0 years before onset (median age 12 years, $42 \%$ females). Assay cutoff was an index of 0.04 , determined as the mean +3 standard deviations of indices from the 91 control sera. Sensitivity and predictive value were calculated as described previously (5). Parallel autoantibody assays used radiolabled icICA512.

To obtain unlabeled icICA512 antigen for competition studies, PCR-generated cDNA sequence for the icICA512 (18) was ligated into the expression vector pZEM $219 \mathrm{~b}$ containing the SV40 promoter (22) and confirmed by sequencing. This vector also constitutively expresses dihydrofolate reductase, allowing methotrexate selection of baby hamster kidney fibroblast transfectants. Cellular icICA512 expression was confirmed by immunocytochemistry (data not shown) using rabbit polyclonal antiserum to ICA512.1 (kindly provided by Dr. D. Rabin) (17). Cells were homognized in $0.25 \%$ Triton X-114 and $10 \mathrm{mM}$ benzamidine in PBS at $\mathrm{pH}$ 7.4. icICA512 concentration was estimated at 7 $\mu \mathrm{g} / \mathrm{ml}$ of cell extract by Western blotting, using as standard recombinant ICA512.1 (kindly provided by Dr. D. Rabin; data not shown). Competition immunoprecipitations used radiolabeled 
icM1851 in the presence of $0.5 \mu \mathrm{g}$ of unlabeled icICA512 per microliter of patient serum. For icM1851 autoantibodies not fully blocked, competitions were repeated at 2.5 -fold greater unlabeled icICA512 with no change in results. Extracts from nontransfected fibroblasts had no effect on icM1851 immunoprecipitation.

PCR-amplified sequences based on intracellular M1851 but containing mutations resulting in the deletion of the C-terminal 27, 80, and 160 amino acids (AA) of the $376 \mathrm{AA}$ intracellular domain, were ligated into pcDNAII, radiolabeled during in vitro expression, and used for duplicate immunoprecipitation assays as described above for intact icM1851.

\section{RESULTS}

The cloning strategy for M1851 in macaque and human is shown in Fig. 1A. Starting with the original macaque library M1851 clone extending from the midextracellular sequence to the $3^{\prime}$ end (sequence 1), successive 5' RACE amplifications yielded sequences 3 through 7 . A longer clone was also identified by screening the library with one of these products, to obtain confirmatory sequence (sequence 2 ). Signal peptide, tribasic site, glycosylation site, transmembrane span, PTPase core sequence, stop codon, and poly-A tail are underlined. The partial human sequence was obtained by PCR using gene-specific primers (sequences 9 to 11 ) and 3' RACE (sequence 8).

The 3,042-nucleotide cDNA sequence and 1,013 residues of primary protein structure of macaque M1851, as well as 2,430 nucleotides and 809 residues of the human sequence, are shown in Fig. 1B. The protein was mildly acidic (predicted pI 5.70) with a calculated molecular mass of 111,190 Da. Where human sequence was available, the human and macaque M1851 sequences were highly homologous (2362/ $2456=96.2 \%$ nucleotide identity; $773 / 817=$ 94.6\% AA identity). As expected, the rat and macaque M1851 sequences were much less similar (75.7\% nucleotide and $69.6 \%$ AA identity).

The 376 AA intracellular domain of human M1851 and the $379 \mathrm{AA}$ intracellular domain of human ICA512 (18) had $73.4 \%$ identity (278/ 379 residues). However, even after optimal alignment, the 612 AA extracellular domain of macaque/human M1851 was in large part distinct $(31.2 \%$ identity, $191 / 612$ residues) from the 576 AA extracellular domain of human ICA512 (Fig. 1C). Greatest similarity in the ex- tracellular region occurred at the N-terminal 100 residues. In addition to the rat and mouse homologues, Genbank searches revealed greatest homology of M1851 with many protein tyrosine phosphatases, including in decreasing similarity, ICA512 (IA2, PTP35, ICA105, IA2a, pheochromocytoma), liver protein tyrosine phosphatase, and leukocyte common antigen (CD45).

Tissue distribution of M1851 was studied using an oligonucleotide probe based on unique sequence just extracellular to the transmembrane domain. Northern analysis using mRNA from 23 human tissues detected strong $5.5-\mathrm{Kb}$ and weaker 3.3-Kb messages in brain, pancreas, and prostate, with still lesser signals in the spinal cord, thyroid, adrenal, and gastrointestinal (GI) tract (Fig. 2). To further define tissue localization, in situ hybridization was performed on macaque pancreas, adrenal gland, and muscle (Fig. 3). Specific signal was demonstrated in pancreatic islets and adrenal medulla, but not muscle. An ICA512 probe and an insulin B-chain probe also hybridized to pancreatic islets, as did the ICA512 probe to adrenal medulla. Unlabeled antisense probes displaced labeled probe binding, treatment of tissue with RNAse A abolished hybridization, and labeled sense oligonucleotides corresponding to each probe showed no hybridization.

The intracellular portion of M1851 was expressed and radiolabeled in vitro in the presence of ${ }^{35} \mathrm{~S}$-methionine. Polyacrylamide gel electrophoresis (PAGE) and autoradiography of the resulting polypeptide revealed a major $46-\mathrm{kDa}$ band and a minor 33-kDa band, both immunoprecipitated by IDDM sera (Fig. 4, left panel). Limited trypsinization of the immunoprecipitated polypeptide followed by SDS-PAGE and autoradiography revealed a $37-\mathrm{kDa}$ fragment from both macaque and human icM1851. This fragment was distinct from the $40-\mathrm{kDa}$ fragment produced by trypsinization of the 379 AA intracellular domain of human ICA512 (Fig. 4, right panel).

Immunopreciptation assays using radiolabeled recombinant intracellular M1851 (icM1851) revealed autoantibodies in 56/183 $(30.6 \%)$ newly diagnosed IDDM patients, 28/60 (46.7\%) first-degree relatives later progressing to clinical diabetes, but only $1 / 91(1.1 \%)$ healthy control subjects. M1851 antibody indices are presented in Fig. 5, left panel, for these three subject groups. For first-degree relatives, this represents a positive predictive value of $58 \%$ at $48 \%$ sensitivity in prediabetes (5). Prediabetics had a significantly higher prevalence of GADab than M1851 when tested more than 5 


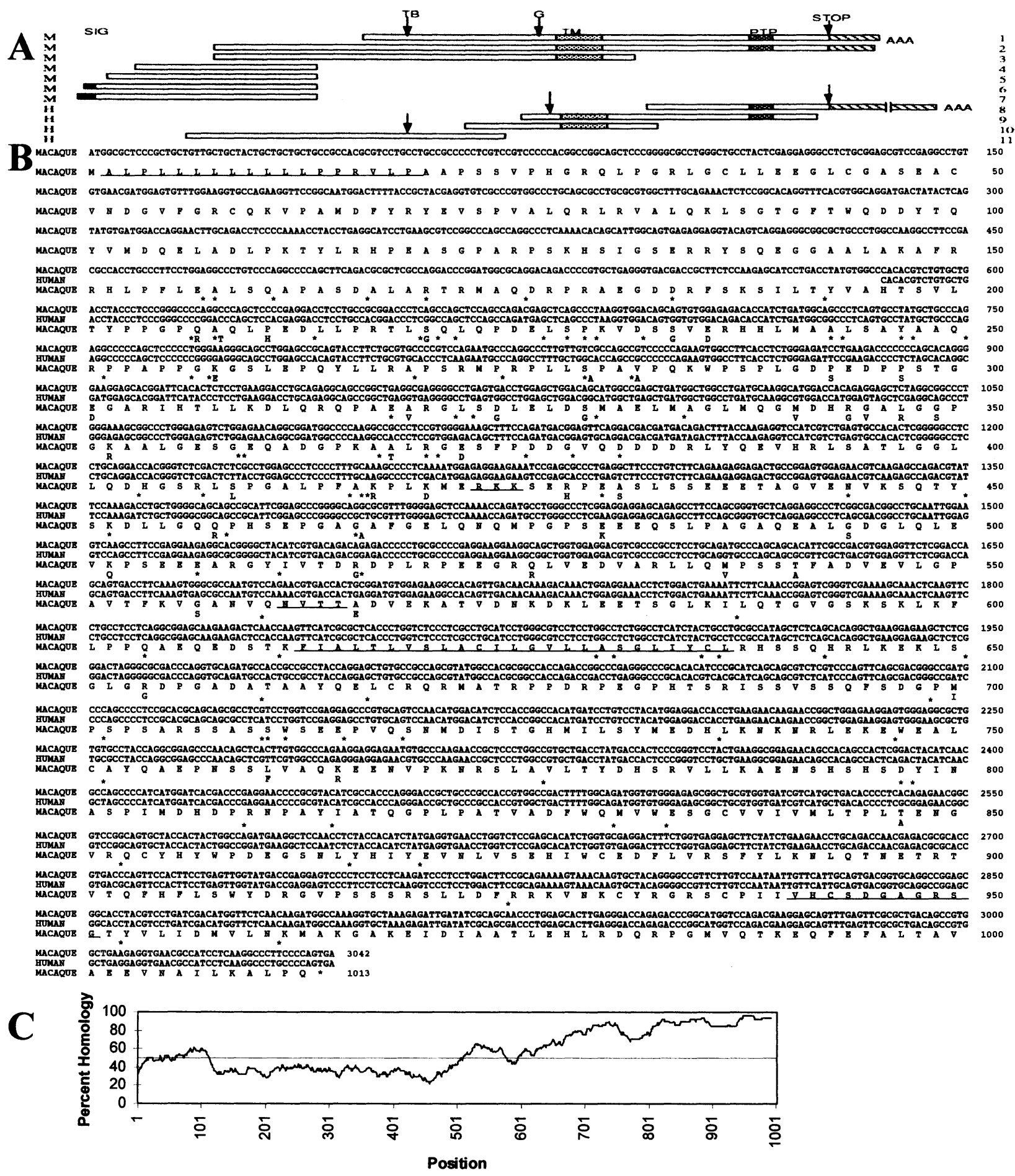

FIG. 1. Cloning strategy, macaque and human sequences, and homology to ICA512/IA2

(A) Cloning strategy for macaque ( $M$, top 7 sequences) and human ( $\mathrm{H}$, bottom 4 sequences) M1851. Specific products described in the text are indicated by numbers to the right in the figure. (B) The 3,042-nucleotide cDNA sequence and 1,014 amino acid primary structure of macaque islet M1851. A partial sequence (2,456 nucleotides) from human M1851 is also aligned. The 11-residue tyrosine phosphatase core sequence, the 25 -residue transmembrane region, an extracellular tribasic site (RKK) and an asparagine glycosylation site (NVTT) of possible posttranslational processing, and the 18-residue signal peptide, are underlined. Asterisks are placed above differences between human and macaque nucleotide sequences, and human amino acids differing from the macaque sequence are noted directly below the corresponding macaque residue. (C) Homology between human ICA512 and human/macaque M1851 proteins. Both identical and conservative changes are allowed. The graph shows homology over a 25 -residue window. The horizontal line represents $50 \%$ homology. 


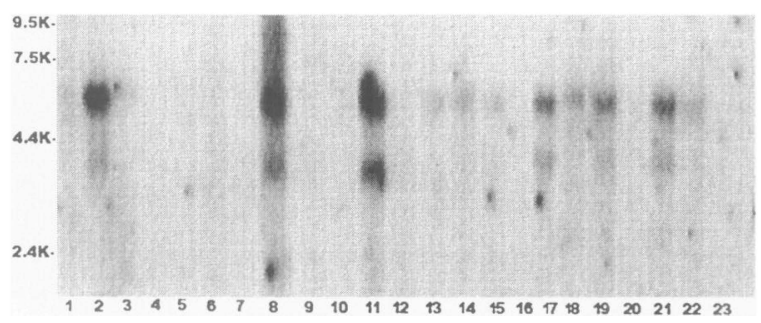

FIG. 2. Northern analysis using an M1851specific oligonucleotide probe on RNA from 20 human tissues

Tissues are as labeled: 1, heart; 2, brain; 3, placenta; 4, lung; 5, liver; 6, skeletal muscle; 7, kidney; 8 , pancreas; 9 , spleen; 10 , thymus; 11 , prostate; 12 , testes; 13, uterus; 14, small intestine; 15, colon; 16, peripheral lymphocytes; 17, stomach; 18, thyroid; 19, spinal cord; 20 , lymph node; 21 , trachea; 22 , adrenal; and 23 , bone marrow. Strong 5.5-kb and weaker 3.3-kb mRNAs (arrows) are present in lanes representing brain, pancreas, and prostate, with lesser signals in spinal cord, adrenal, thyroid, and GI tract.

years before diagnosis (Fig. 5, right panel). However, this difference disappeared completely near the time of diagnosis, which suggests that M1851 autoantibodies appear later in prediabetes than GADab.

Of sera from 153 newly dignosed patients, 83 $(54 \%)$ recognized icICA512 and $48(31 \%)$ recognized icM1851. Only $1 / 48(2 \%)$ sera recognizing icM1851 did not precipitate icICA512, but $35 / 83(42 \%)$ reactive with icICA512 did not precipitate icM1851. Sera reactive to both antigens generally precipitated ICA 512 to a greater extent than M1851, leading to greater calculated autoantibody indices for the former antigen (Fig. 6, left panel). However, it is not known whether greater specific autoantibody concentration or greater affinity accounted for this difference.

In order to test whether M1851 autoantibodies recognized only epitopes shared with ICA512, recombinant icICA512 antigen was expressed in baby hamster kidney fibroblasts. Extracts from these fibroblasts fully blocked icM1851 reactivity in 29/53 icM1851-positive sera tested, while a median of $21.4 \%$ (range 3-55\%) of original immunoreactivity was retained in 24/53 sera (Fig. 6, right panel). Increasing icICA512 competitor concentration did not reduce this residual immunoreactivity in any of these 24 sera, suggesting that unique M1851 epitopes were being recognized. Of these sera, 14/24 had a M1851ab index greater than their ICA512ab index in the left panel of Fig. 6.

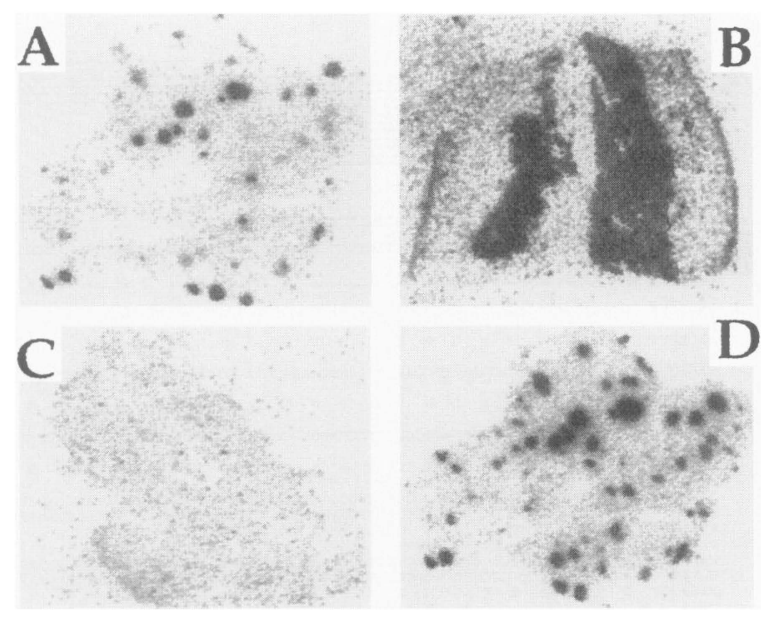

FIG. 3. In situ hybridization in macaque pancreas, adrenal, and muscle using an M1851specific antisense oligonucleotide probe

Strong hybridization was detected in pancreatic islets (A) and adrenal medulla (B) but not in muscle (C). An ICA512-specific antisense probe (D) also hybridized to islets.

Mutants containing C-terminal deletions or mutations from the intact $376 \mathrm{AA}$ icMl 851 were constructed by PCR and expressed for immunoassays as above. Removal of the C-terminal 27 residues decreased reactivity by $32 \%$ from $19 / 53$ to $13 / 53$ IDDM sera. Importantly, antibody level was also greatly decreased, from a median index among positive sera of 0.49 to a median index of 0.04. Removal of the C-terminal $94 \mathrm{AA}$ decreased reactivity further, to $6 / 53$ IDDM sera (11\%), a $68 \%$ decrease from the intact intracellular antigen. Median index decreased to 0.03 . Finally, removal of the C-terminal 159 AA abolished all recognition by the 53 IDDM sera. Whether through primary sequence or complex conformational effects, these results emphasize the importance of the far C-terminal region in M1851 autoantibody epitopes.

\section{DISCUSSION}

Receptor forms of protein tyrosine phosphatases comprise a large superfamily of enzymes with multiple signaling functions regulating cell growth (23), differentiation (24), and adhesion (25). Marked variability in extracellular structure among the transmembrane PTPases is consistent with diverse ligands for adhesion. Intracellular catalytic domains necessarily retain greater homology. Notably, M1851, like ICA512 but unlike most members of the transmembrane PTPase 

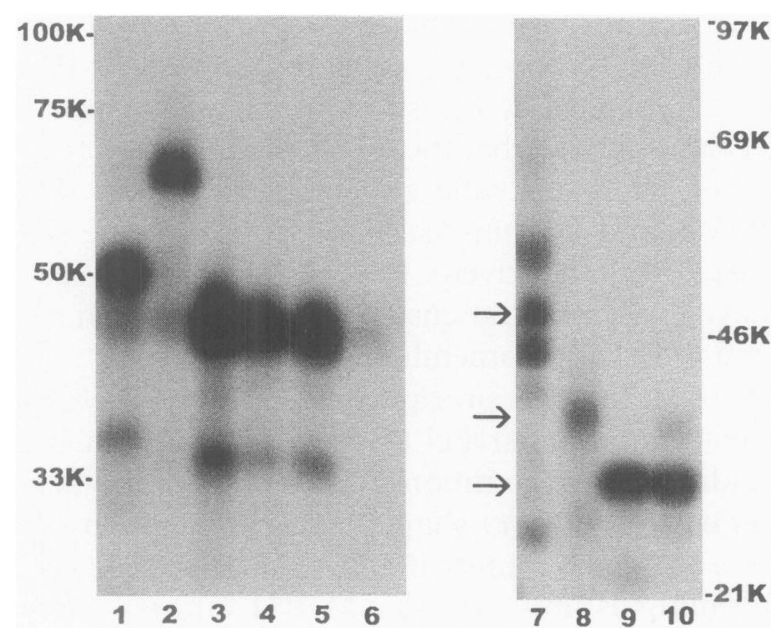

FIG. 4. Polyacrylamide gel electrophoresis and autoradiography of radiolabeled $\beta$-cell antigens Human M1851 (intracellular domain, residues 6371013 ) is shown as total translated protein (lane 3), and after precipitation with rabbit polyclonal antiICA512 (lane 4), with human IDDM serum (lane 5) and with human healthy control serum (lane 6). Human icICA512 (residues 602-979) (lane 1) and whole human GAD65 (residues 1-585) (lane 2) are shown for comparison. GAD65, icICA512, and icM1851 ran at $65 \mathrm{kDa}, 50 \mathrm{kDa}$, and $45 \mathrm{kDa}$, respectively. The major products of limited trypsin digestion of human GAD65, human icICA512, macaque icMl851, and human icM1851 ran at $50 \mathrm{kDa}$, $40 \mathrm{kDa}, 37 \mathrm{kDa}$, and $37 \mathrm{kDa}$, for lanes 7 to 10 , respectively.

family, has a single putative catalytic domain (18). Like ICA512 and LCA (CD45), M1851 also has an Asp-for-Ala substitution in the active site (26). The physiological role of these PTPases in the $\beta$-cell remains to be determined, but the rat homologue of M1851 is associated with insulin secretory granules (26). Neither $\beta$-cell PTPase has yet been proven to be catalytically active (27).

The tissue distribution of M1851 is generally neuroendocrine in nature. Northern analysis here showed strong hybridization to human mRNA from brain and pancreas, and weaker hybridization in spinal cord, thyroid, adrenal, and GI tract. In situ hybridization using macaque tissues further localized expression to pancreatic islets and adrenal medulla. Mouse (24) and rat (26) homologues of M1851 have been recently described. Northern and Western analyses in the rat identified the homologue (termed phogrin) in islets, brain, pituitary, and gastric fundus, as well as insulinoma, glucagonoma, adrenal medullary, and pituitary corticotroph cell lines. The mouse homologue (termed PTP-NP) was identified in developing nervous system and endocrine pan-
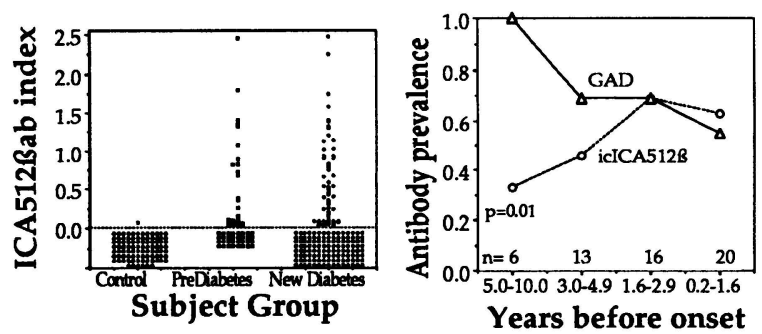

FIG. 5. Recombinant icM1851 autoantibody assay in diabetes and prediabetes

Left: Indices representing relative levels of autoantibodies to recombinant intracellular M1851 (icM1851), which were positive in 56/183 new IDDM patients, 28/60 first-degree relatives later progressing to clinical diabetes, and 1/91 healthy control subjects. The dotted line represents the cutoff for positivity, which is an index of 0.04. Right: Comparison of the prevalence of autoantibodies to GAD and to $M 1851$ versus sampling time before the diagnosis of IDDM. Numbers of subjects in each time range are shown at the bottom of the panel.

creas (24). So far, the only exception to this neuroendocrine distribution is human prostate found positive in our Northern analysis.

Study of the rat homologue (26) detailed a number of basic biochemical features of the protein, most notably an insulin secretory granule membrane location, a dibasic cleavage site corresponding to residues $424-425$ in Fig. 1B, and a putative glycosylation site corresponding to residues 562-565 in Fig. 1B. Evidence of expected
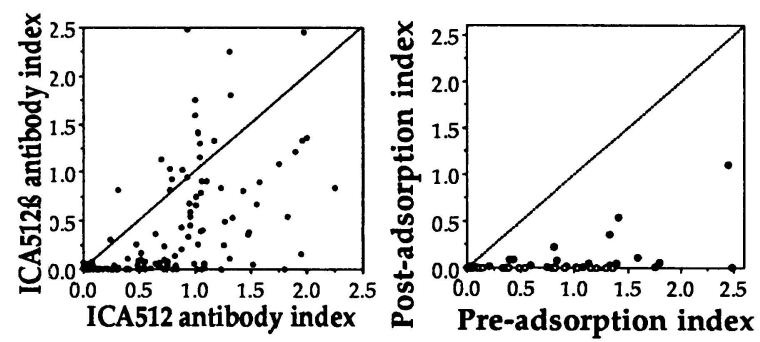

FIG. 6. Comparison of autoantibody epitopes between M1851 and ICA512

Left: Direct comparison of autoantibody indices to icM1851 versus icICA512. Right: Competition of binding of radiolabeled icM1851 by unlabeled recombinant icICA512. For 53 IDDM sera recognizing icM1851, icM1851ab index without competitor is plotted versus the same index in the presence of excess unlabelled icICA512. Reactivity was fully blocked in 29/53 sera (open symbols), while 24/53 retained partial ability to bind icMl 851 (filled symbols). In both panels, the dotted line denotes equal indices. 
sizes following limited proteolysis and endoglycosidase treatment supported the presence of these post-translational modifications. Although only $70 \%$ identical to that of the rodent, the primate M1851 sequence has preserved tribasic and glycosylation sites, and there is no reason to suspect that similar post-translational processing does not occur in humans. Additional common structural features include a cysteine-rich region near the amino terminus (AA 15-60) and multiple potential phosphorylation sites for casein kinase II and protein kinase $\mathrm{C}$ located intracellularly. Including the 1,680-bp human $3^{\prime}$ untranslated region (but not the $5^{\prime}$ untranslated region nor the polyadenosine tail), our estimated human M1851 sequence spans 4,722 nucleotides, compared with the 5.5-Kb mRNA observed by Northern blot. It is not known what accounts for the 2 message sizes $(5.5 \mathrm{~Kb}$ and $3.3 \mathrm{~Kb})$ observed in the human. However, the macaque M1851 homologue from islets had a 3' UTR of only $209 \mathrm{bp}$, which could be consistent with a $3.3-\mathrm{Kb}$ message.

Interestingly, features of both the ICA512 and M1851 PTPases are shared with the other two major targets for autoantibodies in IDDM, GAD and insulin. First, the antigens are at least in part membrane bound $(28,29)$, which may result in more efficient immune recognition (30). Secondly, autoantibody epitopes are found in that part of the protein normally located in the cytosol. Whether these features are present in all autoantigens serving as humoral targets in autoimmunity to $\beta$-cells remains to be determined.

Limited trypsin digestion of the two protein tyrosine phosphatases yields a $40-\mathrm{kDa}$ fragment from ICA512 and a 37-kDa fragment from M1851. This method of trypsin cleavage utilizes antigen bound to human autoantibodies, and yields distinct fragments remaining antibody-bound due to preservation of diabetes-relevant epitopes. Although we have not here proven that these fragments are identical to the tryptic fragments described by Christie et al. (7), a large body of evidence is consistent with this conclusion, which has also been reported by others for trypsin-treated human ICA512 (31) and for trypsin-treated mouse (32) and rat (26) homologues of Ml851. The 37$\mathrm{kDa}$ and 40-kDa tryptic fragments were described by Christie to derive from a faint $64-\mathrm{kDa}$ band distinct from GAD that was soluble only in $8 \mathrm{M}$ urea (8). ICA512 and M1851 contain extracellular (intralumenal) dibasic and tribasic residues, respectively, yielding 592 and 588 residue fragments Cterminal to the putative cleavage site, each still containing a hydrophobic transmembrane se- quence (18). These are of the proper size to account for the poorly soluble and diffuse $64-\mathrm{kDa}$ band described by Christie. Christie and co-workers also showed that the $40-\mathrm{kDa}$ and $37-\mathrm{kDa}$ fragment precursors were glycosylated (31) and that they bound to lectins via $\mathrm{N}$-acetyl glucosamine and mannose, respectively. ICA512 and M1851 each have the proper consensus glycosylation site in the extracellular juxtamembrane sequence.

That immunoprecipitation of these two fragments is often parallel (7) is consistent with our findings of $(a)$ common intracellular amino acid sequences, and $(b)$ shared epitopes between the two antigens in most IDDM sera. That the far C terminus is important for M1851 autoreactivity is consistent with structural requirements described for two of four major icICA512 autoantibody epitopes (33). Taken together, the data that ICA 512 is precipitated by many more sera than M1851, that C-terminal deletions abolish all M1851 reactivity, and that excess ICA512 only partly blocks M1851 reactivity, is consistent with M1851 lacking the juxtamembrane epitope(s) described for ICA5 12 but containing both unique and shared epitopes at the far $C$ terminus.

Although many candidate $\beta$-cell autoantigens have been described for IDDM, remarkably few are actually demonstrated targets of the autoimmune response. Insulin autoantibodies (34) have been shown to be highly predictive, especially in combination with ICA and in the very young. $T$ cell responses to human insulin have also been demonstrated $(35,36)$. A $64-\mathrm{kDa}$ autoantigen (37) identified as glutamate decarboxylase (GAD) is the target of highly predictive autoantibodies (22) and $\mathrm{T}$ cells $(35,38)$. The putative protein tyrosine phosphatase ICA512 (16) is also a target of highly predictive antibodies $(19)$ and T cells $(35,36)$. Other proposed antigens have been less useful. Carboxypeptidase $\mathrm{H}$ antibodies were weakly associated with IDDM (12), ICA69 autoantibodies were not associated with IDDM (12), Imogen 38 is a mitochondrial protein which was not $\beta$-cell specific (39), and Glima 38 reactivity relies on heavy glycosylation and autoantibodies to it were uncommon in diabetes (40). Antibodies to bovine serum albumin (41) and heat shock protein (42) were nonpredictive in diabetes. The 105-kDa RIN antigen (43) and 52-kDa rubella-associated antigen (44) remain elusive, and predictivity has not been confirmed. Data on the reactivity of sulfatides (45) and gangliosides (46) remain limited since these nonprotein antigens are not easily used in immunoassays. That M1851 autoantibodies are 
prevalent in diabetes and prediabetes and that M1851 contains unique autoantibody epitopes not present on ICA512, suggest that this is a new IDDM autoantigen. Given the limited (77\%) amino acid identity in the intracellular domain, unique $\mathrm{T}$ cell epitopes are also possible.

The utility of M1851 autoantibodies in the screening and prediction of IDDM may be limited, since ICA512ab had greater sensitivity whereas only $0.7 \%$ of patients not detected by ICA512ab had M185 lab. Like ICA512ab, M1851 autoantibodies are most prevalent near disease onset, which suggests that $\mathrm{Ml} 85 \mathrm{l}$ is not primary in IDDM pathogenesis. Nevertheless, T lymphocyte help is required to form the high-affinity precipitating autoantibodies we have described. Cellular immunity developing late in prediabetes may be important, for example, in $\beta$-cell killing which has been suggested to occur primarily late in the pathogenesis (47).

Therapies based on such antigens involved late in the pathogenesis may be especially relevant in treating older IDDM patients identified by autoantibody testing at the time of clinical NIDDM diagnosis (22). These late onset IDDM patients are nearly as numerous as classical childhood IDDM $(22,48)$. Late immuno-intervention therapy in these patients may be especially effective $(49,50)$ perhaps because of the slowly progressive nature of their $\beta$-cell destruction. Given the high specificity of HLA-peptide interactions, differences in protein sequence between even homologous domains of ICA512 and M1851 could lead to different $\mathrm{T}$ cell activation by these molecules. Efforts to develop peptides (51) and peptide HLA conjugates (52) for immunotherapy require use of specific amino acid sequences from these or other $\beta$-cell autoantigens.

The presence of unique autoantibody epitopes on M1851, and its general neuroendocrine tissue expression, support M1851 as a new islet autoantigen in IDDM. Its dual roles as a $\beta$-cell insulin secretory granule protein and as a target of the autoimmune response in prediabetes may provide important insights into $\beta$-cell physiology as well as the pathogenesis and therapy of autoimmune diabetes.

\section{ACKNOWLEDGMENTS}

We thank Barbara Snowden, Regina Park, and Rashmi Patel for excellent technical assistance. We also thank Dr. J. P. Palmer for help in procurement of and ICA/IAA measurements on sera used for library screening, Dr. D. Rabin for generous gifts of recombinant intracellular ICA512 and rabbit antiserum to ICA512.1, Dr. D. Baskin for advice on in situ hybridizations, and Dr. G. T. Nepom for assistance in procurement of IDDM sera. This paper was presented in part as a poster at the General Clinical Research Centers Annual Conference, Washington DC, March 1996. WAH is supported by the American Diabetes Assn. by NIH grants to the CRC (RR00037), the DERC (DK17047) and the NW Regional Primate Center (RR00166).

\section{REFERENCES}

1. Atkinson MA, Maclaren NK. (1994) The pathogenesis of insulin-dependent diabetes mellitus. N. Engl. J. Med. 331: 1428-1436.

2. Bottazzo GF, Florin-Christensen A, Doniach D. (1974) Islet cell antibodies in diabetes mellitus with autoimmune polyendocrine deficiency. Lancet ii: 1279-1283.

3. Marshall MO, Hxyer PE, Petersen JS, et al. (1994) Contribution of glutamate decarboxylase antibodies to the reactivity of islet cell cytoplasmic antibodies. J. Autoimmun. 7: 497-508.

4. Bonifacio E, Lampasona V, Genovese S, Ferrari M, Bosi E. (1995) Identification of protein tyrosine phosphatase-like IA2 (islet cell antigen 512) as the IDDM-related $37 / 40 \mathrm{~K}$ autoantigen and a target of ICA. J. Immunol. 155: $5419-5426$.

5. Hagopian W, Sanjeevi C, Kockum I, et al. (1995) Glutamate decarboxylase-, insulinand islet cell-antibodies and HLA typing to detect diabetes in a general population-based study of Swedish children. J. Clin. Invest. 95: 1505-1511.

6. Kolb H. (1994) Immune intervention in type I diabetes mellitus-current clinical and experimental approaches. Exp. Clin. Endocrinol. 102: 269-272.

7. Christie MR, Vohra G, Champagne P, Daneman D, Delovitch TL. (1990) Distinct antibody specificities to a $64-\mathrm{kD}$ islet cell antigen in type 1 diabetes as revealed by trypsin treatment. J. Exp. Med. 172: 789-794.

8. Christie M, Hollands J, Brown T, Michelsen B, Delovitch T. (1993) Detection of pancreatic islet $64,000 \mathrm{Mr}$ autoantigens in insulindependent diabetes distinct from glutamate decarboxylase. J. Clin. Invest. 92: 240-248.

9. Christie MR, Genovese S, Cassidy D, et al. (1994) Antibodies to islet 37K antigen, but not glutamate decarboxylase, discriminate 
rapid progression to IDDM in endocrine autoimmunity. Diabetes 43: 1254-1259.

10. Ongagna J-C, Levy-Marchal C. (1995) Anti$37 \mathrm{~K}$ antibodies are associated with the development of IDDM in individuals with ICA. Diabetologia 38: 370-375.

11. Bingley $\mathrm{P}$, Christie $\mathrm{M}$, Bonifacio $\mathrm{E}$, et al. (1994) Combined analysis of autoantibodies improves prediction of insulin-dependent diabetes in islet cell antibody-positive relatives. Diabetes 43: 1304-1310.

12. Bonifacio E, Genovese S, Braghi S, et al. (1995) Islet autoantibody markers in IDDM: Risk assessment strategies yielding high sensitivity. Diabetologia 38: 816-822.

13. Hagopian W, Karlsen A, Petersen J, et al. (1993) Regulation of glutamic acid decarboxylase (GAD) diabetes autoantigen expression in highly purified isolated islets from Macaca nemestrina. Endocrinology 132: 2674-2681.

14. Komori M, Kikuchi O, Sakuma $\mathrm{T}$, et al. (1992) Molecular cloning of monkey liver cytochrome P-450 cDNAs: Similarity of primary sequences to human cytochromes P-450. Biochim. Biophys. Acta 1171: 141-146.

15. Jelinek L, Lok S, Rosenberg G, et al. (1993) Expression cloning and signaling properties of the rat glucagon receptor. Science 259: 1614-1616.

16. Rabin D, Pleasic S, Palmer-Crocker R, Shapiro J. (1992) Cloning and expression of IDDM-specific human autoantigens. Diabetes 41: $183-186$.

17. Rabin D, Pleasic S, Shapiro J, et al. (1994) Islet cell antigen 512 is a IDDM-specific islet autoantigen related to protein tyrosine phosphatases. J. Immunol. 152: 3183-3188.

18. Lan M, Lu J, Goto Y, Notkins A. (1994) Molecular cloning and identification of a receptor-type protein tyrosine phosphatase, IA-2, from human insulinoma. DNA Cell Biol. 13: $505-514$.

19. Verge C, Gianani R, Kawasaki E, et al. (1996) Prediction of Type 1 diabetes in firstdegree relatives using a combination of insulin, GAD, and ICA512bdc/IA2 autoantibodies. Diabetes 45: 926-933.

20. Karlsen A, Hagopian W, Grubin C, et al. (1991) Cloning and primary structure of a human islet isoform of glutamic acid decarboxylase from chromosome 10. Proc. Natl. Acad. Sci. U.S.A. 88: 8337-8341.

21. Frohman M, Dush M, Martin G. (1988) Rapid production of full-length cDNAs from rare transcripts: Amplification using a single gene-specific oligonucleotide primer. Proc. Natl. Acad. Sci. U.S.A. 85: 8998-9002.

22. Hagopian W, Karlsen A, Gottsäter A, et al. (1993) Quantitative assay using recombinant human islet glutamic acid decarboxylase (GAD-64) shows $64 \mathrm{~K}$ autoantibody positively at onset predicts diabetes type. J. Clin. Invest. 91: 368-374.

23. Dixon J. (1995) Structure and catalytic properties of protein tyrosine phosphatases. Ann. N.Y. Acad. Sci. 766: 18-22.

24. Chiang M-K, Flanagan J. (1996) PTP-NP, a new member of the receptor protein tyrosine phosphatase family, implicated in development of nervous system and pancreatic endocrine cells. Development 122: 22392250.

25. Brady-Kalnay S, Tonks N. (1995) Protein tyrosine phosphatases as adhesion receptors. Curr. Opin. Cell Biol. 7: 650-657.

26. Wasmeier C, Hutton J. (1996) Molecular cloning of phogrin, a protein-tyrosine phosphatase homologue localized to insulin secretory granule membranes. J. Biol. Chem. 271: 8161-8170.

27. Lu J, Notkins A, Lan M. (1994) Isolation, sequence and expression of a novel mouse brain cDNA, mIA-2, and its relatedness to members of the protein tyrosine phosphatase family. Biochem. Biophys. Res. Commun. 204: 930-936.

28. Christgau S, Aanstoot H-J, Schierbeck H, et al. (1992) Membrane anchoring of the autoantigen $\mathrm{GAD}_{65}$ to microvesicles in pancreatic $\beta$-cells by palmitoylation in the $\mathrm{NH}_{2}-$ terminal domain. J. Cell Biol. 118: 309-320.

29. Aguilar-Diosdado M, Parkinson D, Corbett J, et al. (1994) Potential autoantigens in IDDM. Expression of carboxypeptidase- $\mathrm{H}$ and insulin but not glutamate decarboxylase on the betacell surface. Diabetes 43: 418-425.

30. Hartley S, Crosbie J, Brink R, Kantor A, Basten A, Goodnow C. (1991) Elimination from peripheral lymphoid tissues of self-reactive $B$ lymphocytes recognizing membrane-bound antigens. Nature 353: 765-769.

31. Payton M, Hawkes C, Christie M. (1995) Relationship of the $37,000-$ and $40,000-\mathrm{Mr}$ tryptic fragments of islet antigens in IDDM to the protein tyrosine phosphatase-like molecule IA-2 (ICA512). J. Clin. Invest. 96: 15061511.

32. Lu J, Li Q, Xie H, et al. (1996) Identification of a second transmembrane protein tyrosine 
phosphatase, IA $2 \beta$, as an autoantigen in insulin-dependent diabetes mellitus: Precursor of the 37kDA tryptic fragment. Proc. Natl. Acad. Sci. U.S.A. 93: 2307-2311.

33. Lampasona $V$, Bearzatto $M$, Genovese $S$, Bosi E, Ferrari M, Bonifacio E. (1996) Autoantibodies in IDDM recognize distinct cytoplasmic domains in the protein tyrosine phosphatase-like IA2 antigen. J. Immunol. 157: 2707-2711.

34. Palmer JP, Asplin CM, Clemons $P$, et al. (1983) Insulin antibodies in insulin-dependent diabetics before insulin treatment. Science 222: 1337-1339.

35. Hummel M, Durinovic-Bello I, Zeigler A-G. (1996) Relation between cellular and humoral immunity to islet cell antigens in type 1 diabetes. J. Autoimmun. 9: 427-430.

36. Roep B. (1996) T-cell responses to autoantigens in IDDM. The search for the Holy Grail. Diabetes 45: 1147-1156.

37. Baekkeskov S, Nielsen JH, Marner B, Bilde T, Ludvigsson J, Lernmark Å. (1982) Autoantibodies in newly diagnosed diabetic children immunoprecipitate human pancreatic islet cell proteins. Nature 298: 167-169.

38. Atkinson M, Kaufman D, Campbell L, et al. (1992) Response of peripheral blood mononuclear cells to GAD in IDDM. Lancet 339: 458-459.

39. Arden S, Roep B, Neophytou P, et al. (1996) Imogen 38: A novel $38 \mathrm{kD}$ islet mitochondrial autoantigen recognized by $\mathrm{T}$-cells from a newly diagnosed type 1 diabetic patient. J. Clin. Invest. 97: 551-561.

40. Aanstoot H-J, Kang S-M, Kim J, et al. (1996) Identification and characterization of Glima 38 , a glycosylated islet cell membrane antigen, which together with GAD65ab and IA2 marks the early phases of the autoimmune response in type I diabetes. J. Clin. Invest. 97: 2772-2800.

41. Atkinson $M$, Bowman $M, K a o ~ K$, et al. (1993) Lack of immune responsiveness to bovine serum albumin in insulin-dependent diabetes. N. Engl. J. Med. 329: 1853-1858.

42. Atkinson M, Holmes L, Scharp D, Lacy P, Maclaren N. (1991) No evidence for serolog- ical autoimmunity to islet cell heat shock proteins in insulin-dependent diabetes. J. Clin. Invest. 87: 721-724.

43. Thomas N, Ginsberg-Fellner F, McEvoy R. (1990) Strong association between diabetes and displacement of mouse anti-rat insulinoma cell monoclonal antibody by human serum in vitro. Diabetes 39: 1203-1211.

44. Karounas D, Odebunmi T, Hickman S, Stepiek-Biek P. (1992) Sequence determination of the diabetes-associated $52 \mathrm{Kd}$ autoantigen [abstract]. Diabetes 41: 97A.

45. Buschard K, Josefsen K, Rygaard J, Spitalnik S. (1991) Pancreatic islet-cell epitope recognized by an anti-sulphatide monoclonal antibody. APMIS 99: 1151-1156.

46. Dotta F, Dionisi S, Gianani R, et al. (1993) Expression of autoantibodies to the GM-2 islet ganglioside precedes the onset of type 1 diabetes in high risk subjects [abstract]. Diabetologia 36: A24.

47. Shimada A, Charlton B, Taylor-Edwards C, Fathman CG. (1996) $\beta$-cell destruction may be a late consequence of the autoimmune process in nonobese diabetic mice. Diabetes 45: 1063-1067.

48. Harris M, Robbins D. (1994) Prevalence of adult-onset IDDM in the U.S. population. Diabetes Care 17: 1337-1340.

49. Kobayashi T, Nakanishi K, Murase T, Kosaka K. (1996) Small doses of subcutaneous insulin as a strategy for preventing slowly progressive $\beta$-cell failure in ICA-positive patients with clinical features of NIDDM. Diabetes 45: 622-626.

50. Björk E, Berne C, Kämpe O, Wibell L, Oskarsson P, Karlsson FA. (1996) Diazoxide treatment at onset preserves residual insulin secretion in adults with autoimmune diabetes. Diabetes 45: 1427-1430.

51. Fathman C. (1993) Peptides as therapy of autoimmune disease. Diabetes Metab. Rev. 9: 239-244.

52. Nag B, Mukku P, Arimilli S, Phan D, Deshpande S, Winkelhake J. (1994) Antigenic peptide binding to MHC class II molecules at increased peptide concentrations. Mol. Immunol. 31: 1161-1168.

Communicated by D. F. Steiner. Accepted January 16, 1997. 